\section{A rare case of huge congenital parieto-occipital teratoma in a female infant: An unusual site of occurrence}

\author{
Mohammed Kabir Saleh \\ Department of Radiology, Bayero \\ University/Aminu Kano Teaching \\ Hospital, Kano, Nigeria
}

\begin{abstract}
Teratomas are considered the most common congenital tumors located on the dorsal midline and arise from cells derived from more than one germ layer (i.e. ectodermic, endodermis and mesodermic) at different regions of the body. Those in the head and neck regions are considered rare with an incidence of $1-3.5 \%$ of all cases. Imaging provides critical information that helps in formulating differential diagnosis even though, history and physical examination provides critical information in making the diagnosis. A successfully surgically treated case of an usually huge parietooccipital teratoma in a 40 days old girl was presented with emphasis on the importance of imaging in diagnosis and management.
\end{abstract}

\section{Introduction}

Teratomas are rare tumors with a prevalence of approximately $1 / 13,000$, but are however, considered the most common congenital tumors located on the dorsal midline and arises from cells derived from more than one germ layer at different regions of the body due to change in location of the germ cells. ${ }^{1}$ They contain ectodermic, endodermic, and mesodermic tissues.

Teratomas located in the head and neck region are very rare comprising $1-3.5 \%$ of all cases. $^{2}$

More common sites are the sacrococygeal region $(40 \%)$ and the gonads $(40 \%) .^{3}$ Males are reported to have slightly higher incidence (male: female of 2.5:1). ${ }^{4}$

Although clinical history and physical examinations are very important in managing these patients, imaging provides critical information that helps in formulating differential diagnosis.

An infant who presented with a large rare teratoma of the scalp was presented and the importance of imaging in diagnosis and treatment of this lesion was emphasized.

\section{Case Report}

M.A., a 40 days-old girl was brought to the Paediatric Out-patient Department of Aminu Kano Teaching Hospital by her mother on account of a mass on the right side of the head which extended to the back of the head. The mass was said to have been noticed during routine prenatal sonography at second trimester of gestation at the referral clinic where antenatal care was booked. The mother who has four other healthy children with no history of similar mass at birth attended antenatal visits regularly in the course of the pregnancy of this patient. She had no history of consumption of alcohol or non prescribed medications during pregnancy.

Delivery of the child was via a caesarian section on account of obstructed labour due to dystocia. The child was said to have cried immediately at birth and did well since delivery. The mass on the right side of head was however said to have increased in size from its initial size at birth.

On physical examination, the general condition of the child was good. She was alert with an interest in the environment. There was a soft tissue, mobile, cystic mass overlying the right parieto-occipital region of the skull with an intact skin covering. It measured $17 \times 24 \mathrm{~cm}$ in size. No neurological deficits were noted on neurological assessment. Abdominal, cardio-respiratory and musculoskeletal systemic physical examinations were essentially normal. No swelling was seen in other parts of the body. Full blood count and differentials including PCV, Urea and creatinine, chest radiographs, abdominal ultrasound scan and transcranial cranial ultrasound scan of the brain were found to be within normal limits. An initial diagnosis of cystic hygroma was made. Differential diagnoses considered included giant teratoma, encephalocele, lipoma, dermoid cyst, dermal cysts and hemangioma.

A plain radiograph of the skull (Figures 1 and 2) showed a large, fairly oval shaped mass of soft tissue density with regular outline and margins and internal calcifications in the right parieto-occipital region. No associated adjacent bony destruction or evidence to suggest intracranial extension of the mass was seen. Ultrasonography of the mass (Figure 3) revealed a mixed echogenic appearance with both solid and cystic components. Focal areas of calcifications were noted within it. Doppler interrogation showed absent blood flow within the mass suggestive of hypovascular lesion. These findings in conjunction with those of clinical examination were suggestive of a benign soft tissue mass likely teratoma. She
Correspondence: Mohammed Kabir Saleh, Department of Radiology, Bayero University, Aminu Kano Teaching Hospital, Kano, Nigeria.

Tel.: +2348037872982

E-mail: mk_saleh@yahoo.com

Key words: Teratoma, Parieto-occipital, Congenital Huge, Rare.

Acknowledgement: I wish to acknowledge the assistance of the radiographer, Mr Nafi'u Muhammad Ahmad for taking the skull radiographs of the patient. Same is also extended to Dr Hassan Isma'il and Prof. Anyanwu L.J.C. for the skillful surgical removal of the lesion and also management of the patient and also $\mathrm{Dr}$ Ali Bala for the confirmatory histopathogical diagnosis on the excised tiusse.

Conflict of interest: The author declares no conflict of interest.

Availability of data and materials: Not applicable.

Ethics approval and consent to participate: This was obtained from the ethics and research committee of the Aminu Kano Teaching Hospital, Kano, Nigeria and the patient identity is well protected

Received for publication: 27 August 2019.

Revision received: 30 September 2019.

Accepted for publication: 15 July 2020.

This work is licensed under a Creative Commons Attribution NonCommercial 4.0 License (CC BY-NC 4.0).

${ }^{\circ}$ Copyright: the Author(s), 2019

Licensee PAGEPress, Italy

Pyramid Journal of Medicine 2020; 3:61

doi:10.4081/pjm.2020.61

had a total surgical excision and histology of the mass, which on gross inspection of its content revealed hairy and finger-like structures embedded in a gelatinous fluid. Primary skin closure was done after securing haemostasis. The report at histology confirmed a grade II-III immature teratoma. The patient had uneventful post-operative recovery. She was discharged from the hospital one week post-op and was followed-up in the pediatric outpatient clinic. At six months of regular follow-up, the wound has healed well with no evidence of tumor recurrence.

\section{Discussion}

Teratomas commonly occur in the sacrococcygeal region in children. They may also occur in the retroperitoneal, anterior mediastinum and thymus regions, including the pharynx, brain, orbit, pelvis 
and spinal regions, gonads, and subcutaneous tissues. $^{2}$

Head and neck teratomas are rare conditions with a few reports in the literature. In a comprehensive review ${ }^{3}$ conducted between 1966 and 2005, 10 teratoma patients were identified who had a midline teratoma on the back of the neck. The patient in this report had a parieto-occipital located lesion.

Males are reported to have slightly higher incidence (male: female of 2.5:1). ${ }^{4}$ The index case is however seen in a female infant.

Histopathologically, teratomas are typically divided into three groups (mature, immature, and malignant). Mature teratomas contain well differentiated cells, while immature teratomas contain primitive structures that are not adequately differentiated. Teratomas that contain malignant component are classified as a malignant teratomas. ${ }^{2}$ This patient had the benign form.

Both mature and immature forms usually contain tissues from all three germ layers, including skeletal muscle, cartilage, bone, bronchial epithelium, gut epithelium, and neural tissue. According to O'Connor and Norris, mature teratoma is grade- 0 , immature teratoma is classified as grade- 3 , based on the proportion of its immature elements and the mitotic rate. ${ }^{5}$ Histopathological examination of specimen from the present patient contained immature elements (grade-3).

Imaging is very relevant in evaluation of children with cranial masses both in pre and post natal life as teratomas on the head and neck regions of the newborn can be easily confused with other causes such as encephalocele.

Congenital teratomas are often easily recognized during pregnancy by Ultrasound scan and Magnetic Resonance Imaging studies. ${ }^{6}$ Ultrasound is non-invasive and safe. At prenatal Ultrasound, the diagnosis of teratoma should be considered for a complex cranial mass with calcifications. Mostly they are seen on post natal examination as large, heterogeneously echogenic masses with cystic and solid components as well as presence of characteristic calcific elements in it. Sonographic examination of the mass in this patient showed similar features (Figure 3).

Cranial encephalocele closely resembles this condition and Ultrasound diagnosis is based on the recognition of a cystic (meningo-encephalocele) or complex (meningo-encephalocele) mass of variable size protruding through a skull defect, ${ }^{7}$ often localized in the occipital region. The mass usually lacks calcification as in teratoma. There may be associated hydro-

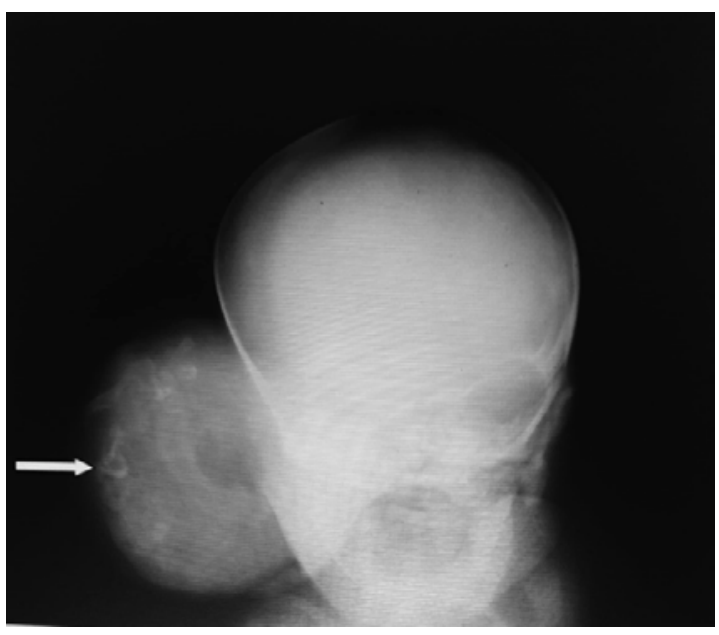

Figure 1. Frontal skull radiograph of the infant showing a large, well defined, fairly oval mass of soft tissue density with some calcific elements within it (white arrow). The mass overlies the right parietal bone displacing the pinna anteriorly. No adjacent bone destruction is seen.

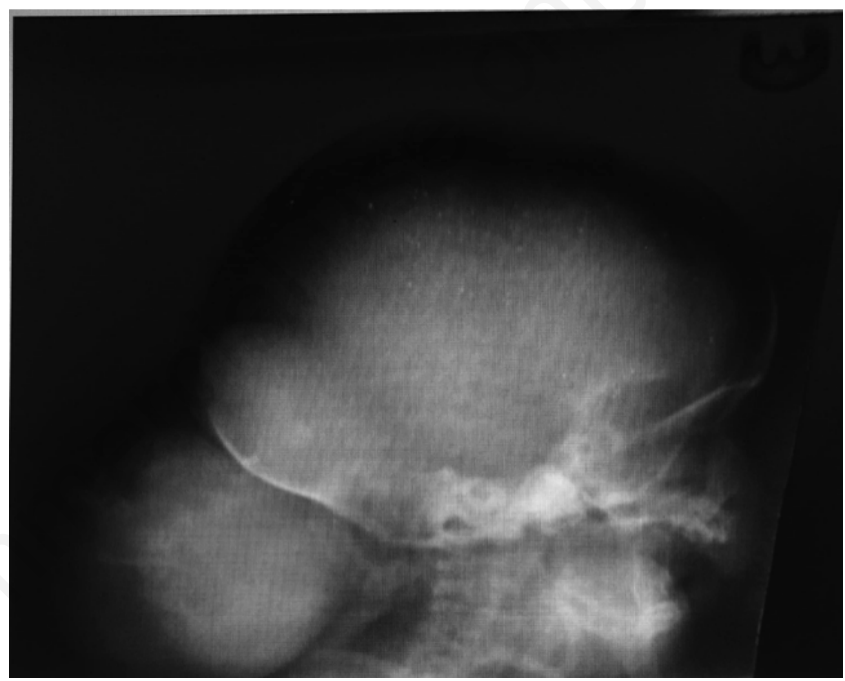

Figure 2. Lateral skull radiograph of the infant showing the occipital component of the soft tissue mass with internal calcific elements. The adjacent occipital bone is intact.

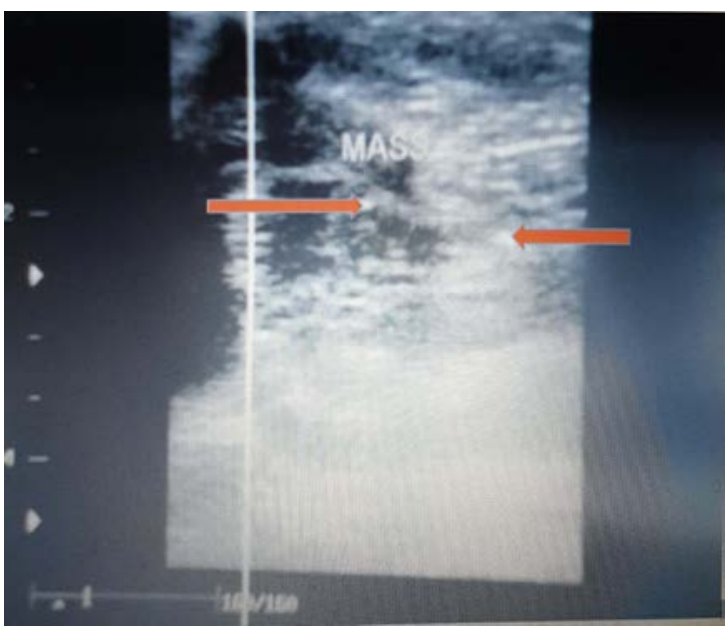

Figure 3. High resolution Ultrasonographic image showing the mass as heterogeneously echogenic in nature with both solid and cystic components as well as focal areas of calcifications (red arrows) in it. 
cephalus and a spina bifida. The index patient did not have any of these abnormalities.

The plain skull radiograph demonstrates a soft tissue mass overlying the involved area of skull vault with calcific foci. There is usually preservation of the adjacent cranial vault. In the index case, the skull radiograph (Figures 1 and 2) demonstrated characteristic calcific structures within the mass with intact adjacent skull bone. ${ }^{4}$

Computed Tomography (CT) and Magnetic Resonance Imaging (MRI) are valuable diagnostic procedures in showing the extracranial localization of the mass and ruling out intracranial extension. ${ }^{4}$ These were not done for this patient due to financial constraints.

Teratomas show cystic and solid areas and areas of fat density at CT and MRI. ${ }^{2}$ The typical T1- and T2-weighted imaging appearance is a large heterogenous mass with cystic component. No apparent difference between mature and immature teratomas may be identified. ${ }^{1}$ Although MR imaging is poor for detecting small calcifications, CT has demonstrated regions of calcification in most teratomas.

In addition to cranial encephalocele, other differential diagnoses of teratomas of the head and neck may include hemangiomas, cystic hygromas, lymphangiomas, lipomas, dermal cysts, vascular malformations, and cutaneous cysts. ${ }^{4}$
The treatment of teratomas consists of early and complete resection of the tumor mass as was done for this patient. Surgical intervention can prevent the risk of malignant transformation. ${ }^{8}$

The prognosis is excellent after total excision in scalp teratomas such as in this case. $^{9}$

\section{Conclusions}

A rare case of right parieto-occipital teratoma in a 40 days-old female child was presented who had imaging and histological diagnosis. She had a total surgical excision of the tumor with no post-operative complications and uneventful follow up.

\section{References}

1. Bare JB, Abramowsky CR, Hayes LL, Shehata BM. Congenital immature teratoma of the central nervous system: Three case reports with literature review. Fetal Ped Path 2007;26:109-18.

2. Dadmehr M, Nejat F, Ansari S, Habibi Z. Ruptured occipito-cervical teratoma mimicking an upper cervical myelomeningocele. J Neurosurg 2006;104:360-1.

3. Shah A, Latoo S, Ahmed I, Malik AH. Head and neck teratomas. J Maxillofac
Oral Surg 2009;8:60-3.

4. Grimme JD, Camacho DLA, Spampinato MV, Castillo M. Teratomas of the Brain and Head and Neck. Departmental case presentations, Department of Radiology, University of North Carolina 2004.

5. Mann JR. Mature and immature extracranial teratomas in children. The UK Children's cancer study group Experience. J Clin Oncol 2008;26: 3590-97.

6. Carstensen H, Juhler M, Bøgeskov L, Laursen H. A report of nine newborns with congenital brain tumours. Childs Nerv Syst 2006;22:1427-31.

7. Vasiljevic B, Gojnic M, MaglajlicDjukic S. Ultrasound Diagnosis of Congenital Brain Anomalies, Congenital Anomalies - Case Studies and Mechanisms. Sutcliffe A (Ed.), London: InTech Publishers; 2012; $102-$ 3.

8. Turgut M, Meteoğlu I. Mature teratoma associated with an interparietal encephalocele. J Neurosurg 2007;106: 305-7.

9. Prevedello DM, Kassam AB, Carrau RL, et al. Transpalatal endoscopic endonasal resection of a giant epignathus skull base teratoma in a newborn case report. J Neurosurg 2007;107:26671 